\title{
Epithelial to mesenchymal transition markers expressed in circulating tumour cells of early and metastatic breast cancer patients
}

\author{
Galatea Kallergi $^{1 *}$, Maria A Papadaki ${ }^{1 \dagger}$, Eleni Politaki ${ }^{1}$, Dimitris Mavroudis ${ }^{1,2}$, Vassilis Georgoulias $^{1,2}$ and
} Sophia Agelaki ${ }^{1,2}$

\begin{abstract}
Introduction: Epithelial to mesenchymal transition (EMT) is considered an essential process in the metastatic cascade. EMT is characterised by upregulation of vimentin, Twist, Snail, Slug and Sip1 among others. Metastasis is also associated with the presence of circulating tumour cells (CTCS) and disseminated tumour cells in the blood and bone marrow, respectively, of breast cancer patients, but the expression of EMT markers in these cells has not been reported so far.

Methods: The expression of Twist and vimentin in CTCS of 25 metastatic and 25 early breast cancer patients was investigated by using double-immunofluorescence experiments in isolated peripheral blood mononuclear cell cytospins using anti-cytokeratin (anti-CK) anti-mouse (A45-B/B3) and anti-Twist or anti-vimentin anti-rabbit antibodies.

Results: Among early breast cancer patients, vimentin-and Twist-expressing $\mathrm{CK}^{+} \mathrm{CTC}$ were identified in $77 \%$ and $73 \%$ of the patients, respectively, and in 100\% of the patients with metastatic breast cancer for both markers $(P=0.004$ and $P=0.037$, respectively). Among patients with early disease, $56 \%$ and $53 \%$ of the $\mathrm{CK}^{+}$CTCs were double-stained with vimentin and Twist, and the corresponding values for metastatic patients were $74 \%$ and $97 \%$, respectively $(P=0.005$ and $P=0.0001$, respectively). The median expression of $\mathrm{CK}^{+}$vimentin ${ }^{+}$and $\mathrm{CK}^{+} \mathrm{T}_{\text {wist }}{ }^{+}$cells per patient in metastatic patients was $98 \%$ and $100 \%$, and in an adjuvant chemotherapy setting the corresponding numbers were $56 \%$ and 40.6\%, respectively. Triple-staining experiments revealed that all $\mathrm{CK}^{+} \mathrm{Tw}_{\text {wist }}{ }^{+}$or $\mathrm{CK}^{+}$vimentin ${ }^{+}$cells were also CD45confirming their epithelial origin. Immunomagnetic separation of CTCs and triple-immunofluorescence with anti-CK anti-Twist/anti-vimentin antibodies demonstrated that both mesenchymal markers could be coexpressed in the same $\mathrm{CK}^{+}$cell, since $64 \%$ of the total identified CTCs were triple-stained. There was a significant correlation $(P=0.005)$ between the number of CTCs expressing Twist and vimentin within the same setting.
\end{abstract}

Conclusions: CTCs expressing Twist and vimentin, suggestive of EMT, are identified in patients with breast cancer. The high incidence of these cells in patients with metastatic disease compared to early stage breast cancer strongly supports the notion that EMT is involved in the metastatic potential of CTCs.

\section{Introduction}

Metastasis is associated with the presence of peripheral blood circulating tumour cells (CTCs) and bone marrow disseminated tumour cells (DTCs) in patients with breast cancer [1,2]. In fact the presence of CTCs before the initiation and after the completion of adjuvant

\footnotetext{
* Correspondence: kalergi@med.uoc.gr

+ Contributed equally

'Laboratory of Tumor Cell Biology, University of Crete, Voutes 71110, Heraklion, Crete, Greece

Full list of author information is available at the end of the article
}

chemotherapy is associated with poor clinical outcome [3-5]. In metastatic breast cancer, the assessment of CTCs before and shortly after the initiation of chemotherapy is also predictive of progression-free and overall survival [6,7], and prognosis seems to depend on the detection of CTCs rather than DTCs [8]. The presence of chromosomal alterations confirmed the malignant nature of CTCs $[9,10]$. Nevertheless, only some of them are capable of promoting metastasis [11]. Therefore, further molecular characterisation of CTCs is

\section{Biomed Central}


essential to understanding their metastatic potential, as well as for the identification of additional markers related to patients' prognosis.

The metastatic process consists of distinct steps, including tumour growth, angiogenesis, tumour cell detachment, epithelial to mesenchymal transition (EMT), intravasation, survival within blood and lymphatic vessels and embolisation, extravasation, mesenchymal to epithelial transition, formation of micrometastasis and, finally, growth of macrometastasis [12]. EMT is a process whereby epithelial cells lose their epithelial characteristics and acquire a mesenchymal phenotype. EMT increases the metastatic and invasive potential of these cells [13]. Downregulation of epithelial markers such as cytokeratin and E-cadherin and upregulation of mesenchymal markers such as vimentin, $\mathrm{N}$-cadherin and cadherin 11 characterise the EMT process. Usually, inhibition of E-cadherin expression leads to induction of $\mathrm{N}$ cadherin expression, which has been associated with tumour invasiveness [14-16]. Transforming growth factor $\beta$ as well as transcription factors such as Twist, Snail, Slug and Sip1 have a regulatory role in EMT.

Twist is a transcriptional repressor of the E-cadherin gene $[12,17]$. Increased expression of Twist has been demonstrated in many types of tumour cells, such as melanoma, osteosarcoma, T cells (Sézary syndrome) and gastric, prostate and breast cancer [12,18-23]. The gene expression profile of immunomagnetically isolated DTCs has shown elevated expression of Twist in the enriched fragment compared to that in healthy volunteers [24,25]. Twist expression in breast cancer cells has been shown to result in resistance to paclitaxel through binding to Akt promoter and enhancement of its transcriptional activity [26], as well as resistance to other microtubuletargeting agents such as vincristine $[27,28]$. Upregulation of Twist in cancer cells increases vascular endothelial growth factor (VEGF) gene expression [21,29], while hypoxia-inducible factor $1 \alpha(\mathrm{HIF}-1 \alpha)$ regulates the expression of Twist by binding directly to the hypoxia response element in the Twist proximal promoter $[30,31]$. We have recently shown that VEGF and HIF$1 \alpha$, as well as phosphorylated Akt, are expressed in CTCs of most metastatic breast cancer patients [32,33]; therefore, it was interesting to further investigate the expression of Twist in CTCs of breast cancer patients.

Vimentin is an intermediate filament normally expressed in mesenchymal cells and is involved in the migration of epithelial cells during development [34]. The expression of vimentin in cancer cells is believed to enhance migration and invasiveness [35]. Expression of vimentin is characteristic of epithelial cells undergoing the EMT process and is related to reduced expression of E-cadherin and upregulation of $\mathrm{N}$-cadherin $[16,36]$, while increased expression of vimentin in breast carcinomas is correlated with poor prognosis [37]. Moreover, the simultaneous expression of vimentin and cytokeratin in tumour cells is associated with poorer survival in breast cancer patients [38]. Recent studies have demonstrated that vimentin is expressed in DTCs of breast cancer patients and tumour cell lines $[39,40]$; however, there have been no studies in which the expression of both EMT markers (Twist and vimentin) was evaluated in CTCs. Therefore, the aim of the present study was to investigate the expression of these molecules in CTCs of patients with early and metastatic breast cancer.

\section{Materials and methods}

\section{Patient samples and cytospin preparation}

A longitudinal trial for the study of micrometastatic disease in breast cancer has been underway in our institution since 1996. Peripheral blood samples are obtained from patients who provide their written informed consent as part of the routine evaluation before the initiation of and at the end of adjuvant treatment, as well as prior to and after the completion of each chemotherapy line in patients with metastatic disease. Frozen RNA samples and peripheral blood mononuclear cell (PBMC) cytospins are prepared simultaneously and stored at $-80^{\circ}$ $C$ until use. In the current trial, samples taken from patients with adjuvant or metastatic breast cancer were screened for cytokeratin (CK)-19 mRNA expression [41,42] by RT-PCR. Fifty CK-19 mRNA-positive patients (25 with early stage breast cancer and 25 with metastatic breast cancer) were enrolled in the present study. We used archived slides from these patients because we wanted to have the same blood samples for real-time RT-PCR and immunocytochemistry. Ten healthy female blood donors were also included as a control group. All blood samples were obtained at the middle of venipuncture after the first $5 \mathrm{~mL}$ of blood were discarded. These precautions were undertaken to avoid contamination of the blood samples with epithelial cells from the skin during sample collection. All patients and healthy volunteers gave their written informed consent to participate in the study, which has been approved by the Ethics and Scientific Committees of our institution.

The volume of blood drawn from each patient was 20 $\mathrm{mL}$ each for immunofluorescence and RT-PCR experiments and $20 \mathrm{~mL}$ for immunomagnetic isolation. PBMCs were isolated by Ficoll-Hypaque density gradient centrifugation $(d=1,077 \mathrm{~g} / \mathrm{mol})$ at $1,800 \mathrm{rpm}$ for 30 minutes. PBMCs were washed three times with PBS and centrifuged at $1500 \mathrm{rpm}$ for 10 minutes. Aliquots of 250,000 cells were cytocentrifuged at 2,000 rpm for 2 minutes on glass slides. Cytospins were dried up and stored at $-80^{\circ} \mathrm{C}$. Four to five slides from each patient 
were used for staining experiments, so $1 \times 10^{6}$ PBMCs per patient were scanned.

\section{Cell cultures}

In control experiments, we used the human cervical adenocarcinoma cell line HeLa. HeLa cells express Twist and vimentin and have been proposed as a positive control by the antibody's data sheet. The HeLa adenocarcinoma cells (American Type Culture Collection, Manassas, VA, USA) were cultured in 1:1 ( $\mathrm{vol} / \mathrm{vol})$ DMEM (Gibco-BRL, Grand Island, NY) supplemented with $10 \%$ foetal bovine serum (FBS) (Gibco-BRL), 2 mmol L-glutamine (Gibco-BRL) and $50 \mathrm{mg} / \mathrm{mL}$ penicillin/streptomycin (Gibco-BRL). Cells were maintained in a humidified atmosphere of $5 \% \mathrm{CO}_{2}$ and $95 \%$ room air. Subcultivation of all cell lines was performed using $0.25 \%$ trypsin and $5 \mathrm{mmol}$ ethylenediaminetetraacetic acid (EDTA) (Gibco-BRL). All experiments were performed during the logarithmic growth phase 15 to 20 hours prior to the experiments. HeLa cells were spiked in blood obtained from healthy volunteers, and cytospins were prepared afterward with Ficoll-Hypaque density gradient centrifugation as per patients' samples.

\section{Double-immunofluorescence confocal laser-scanning and ARIOL scanning microscopy}

The presence of CK-positive cells in PBMC cytospin preparations was investigated using the mouse $\mathrm{A} 45-\mathrm{B} /$ B3 antibody (detecting CK8, CK18 and CK19) (Micromet, Munich, Germany). Control experiments for the sensitivity and the specificity of this antibody have been reported previously $[32,33,43]$. The cytomorphological criteria proposed by Meng et al. [44] (for example, high nuclear/cytoplasmic ratio, larger cells than white blood cells) were used to characterise a CK-positive cell as a CTC.

Cytospins from the same patients were double-stained for Twist/CK (Abcam, Cambridge, UK) and vimentin/ CK (Santa Cruz Biotechnology, Santa Cruz, CA, USA) in double-staining experiments [43]. Specific staining can easily be distinguished by double-immunofluorescence because of the differential intracellular distribution of the examined molecules compared to nonspecific staining as reported by Fehm et al. [45]. For doublestaining experiments, PBMC cytospins were fixed with $3 \%$ paraformaldehyde. Permeabilisation of the cell membrane was performed with $0.5 \%$ Triton for $10 \mathrm{~min}$ and blocking with PBS/1\% BSA overnight. Subsequently, slides were stained for cytokeratin with the A45-B/B3 anti-mouse antibody along with the corresponding secondary fluorescein isothiocyanate (FITC) fluorochrome. Slides were then stained with Twist or vimentin antirabbit antibodies and afterward with the corresponding anti-rabbit secondary antibodies for 45 minutes.
Negative controls were performed for all the primary antibodies by omitting the corresponding primary antibody and adding the secondary immunoglobulin $G$ (IgG) isotype antibody. Finally, 4',6-diamidino-2-phenylindole (DAPI) antifade reagent (Invitrogen, Carlsbad, CA, USA) was added to each sample for nuclear staining. Slides were analysed using a confocal laser-scanning microscope (Leica Lasertechnik, Heidelberg, Germany) and the ARIOL CTC automated image analysis system (Genetix, New Milton, Hampshire, UK).

\section{Triple immunofluorescence}

Triple-immunofluorescence for CK/Twist/CD45, CK/ vimentin/CD45 and CK/Twist/vimentin was also performed in samples processed by immunomagnetic separation to be enriched in CTCs. Cells were initially fixed using 4\% formaldehyde for 15 minutes at room temperature. Permeabilisation was achieved with $0.1 \%$ Triton X-100 for 5 minutes at room temperature. After blocking with PBS supplemented with 10\% (vol/vol) FBS for 30 minutes, cells were incubated with the corresponding antibodies for 45 minutes each. Zenon technology (FITC-conjugated IgG1 antibody) (Molecular Probes/Invitrogen, Carlsbad, CA, USA) was used for CK detection with the A45-B/B3 antibody. Zenon antibodies were prepared within 30 minutes of being used.

Twist was detected using anti-mouse antibody (Abcam) labelled with Alexa Fluor 633 (Molecular Probes/Invitrogen) or Twist anti-rabbit (Cell Signaling Technology, Boston, MA, USA) labelled with Alexa Fluor 555 (Molecular Probes, Carlsbad, CA, USA). Positive and negative controls for Twist anti-rabbit are shown in Figure 1A, while positive and negative controls for the Twist anti-mouse antibody are presented in Additional file 1. Vimentin was detected using anti-rabbit antibody (Santa Cruz Biotechnology) labelled with Alexa Fluor 555, and positive and negative controls are shown in Additional file 1. CD45 was detected with an anti-mouse antibody (Dako, Carpinteria, CA, USA) labelled with Alexa Fluor 633. Cells incubated with the different antibodies were postfixed with $4 \%$ ( $\mathrm{vol} / \mathrm{vol}$ ) formaldehyde in PBS for 15 minutes at room temperature. Finally, cells were stained with DAPI conjugated with antifade reagent.

\section{Immunomagnetic separation of CTCs}

A negative selection procedure was used for the isolation of CTCs according to the method described by Naume et al. [46]. A quantity of $100 \mu \mathrm{L}$ of Dynal CELLection beads (Invitrogen) coated with anti-CD45 monoclonal antibody were added to $1 \times 10^{7 \mathrm{P}} / \mathrm{mL}$ PBMCs in PBS/0.1\% BSA/2 mM EDTA. After incubation for 30 minutes at $4^{\circ} \mathrm{C}$, the supernatant was transferred into FBS-coated tubes and cells were 


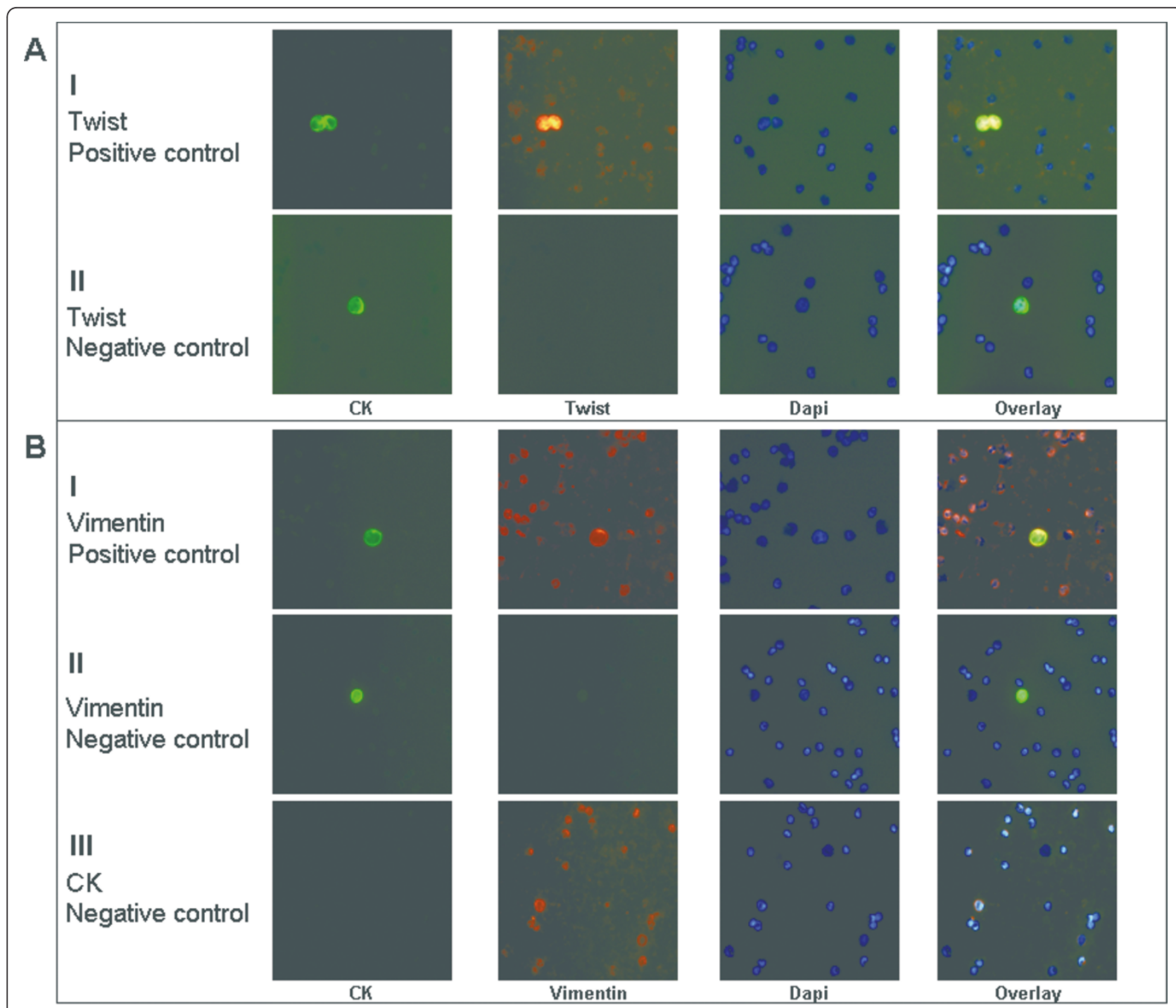

Figure 1 Twist and vimentin expression in HeLa cells spiked in blood of normal volunteers. ARIOL system images of HeLa cells spiked in the blood of normal volunteers. (A) Row I: Positive control for Twist. HeLa cells were stained with pan-CK A45-B/B3 antibody/secondary FITC anti-mouse antibody (green)/Twist anti-rabbit antibody/Alexa Fluor 555 anti-rabbit antibody (orange). Row II: Negative control for Twist. Cells were stained with pan-CK A45-B/B3 antibody/FITC anti-mouse (green) and Alexa Fluor 555 lgG isotype antibody. Cell nuclei were stained with DAPI (blue). Original magnification, x400. (B) Row I: Positive control for vimentin. Cells were stained with pan-CK A45-B/B3 antibody/FITC antimouse antibody (green)/vimentin anti-rabbit antibody/Alexa Fluor 555 anti-rabbit antibody (orange). Row II: Negative control for vimentin. Cells were stained with pan-CK A45-B/B3 antibody/FITC anti-mouse antibody (green) and Alexa Fluor 555 lgG isotype antibody. Row III: Negative control for pan-CK. Cells were stained with FITC IgG isotype antibody/vimentin anti-rabbit antibody/Alexa Fluor 555 anti-rabbit antibody (orange). Cell nuclei were stained with DAPI (blue). Original magnification, $x 400$. ARIOL system = automated image analysis system; CK $=$ cytokeratin; FITC = fluorescein isothiocyanate; HeLa = patient with cervical adenocarcinoma from which the cell line was derived; lgG = immunoglobulin G; DAPI $=$ 4,6-diamidino-2-phenylindole.

cytocentrifuged at $2,000 \mathrm{rpm}$ for 2 minutes on glass slides. The same number of cells was centrifuged at $1,500 \mathrm{rpm}$ for 5 minutes, and the pellet was stored at $-80^{\circ} \mathrm{C}$ for RNA extraction. The specificity and the sensitivity of this method has been described previously [33]. CD45 immunomagnetic depletion was performed for the purpose of triple-staining experiments only to enrich the samples with CTCs.

\section{Results}

Twist and vimentin expression in HeLa cells and PBMCs of normal blood donors

HeLa cell cytospins spiked in blood from healthy volunteers and processed as per patient samples were used as positive controls for the detection of Twist and vimentin. Positive and negative controls for Twist, vimentin and $\mathrm{CK}$ are shown in Figure 1. 
Twist and vimentin expression was subsequently investigated in PBMC cytospins from 10 healthy blood donors. Both molecules were found to be expressed spontaneously in PBMCs, but there were no doublepositive cells $\left(\mathrm{CK}^{+} \mathrm{Twist}^{+}\right.$or $\mathrm{CK}^{+}$vimentin $\left.{ }^{+}\right)$in healthy volunteers.

Twist and vimentin expression on CTCs of early stage and metastatic breast cancer patients

Twenty-five patients with metastatic breast cancer and twenty-five patients with early breast cancer who had detectable CK-19 mRNA-positive cells by RT-PCR were enrolled in this study. The concordance between RTPCR and immunocytochemistry was $100 \%$ in metastatic breast cancer patients and $88 \%$ in early breast cancer patients. The clinicopathological characteristics of these patients are presented in Table 1.

We investigated the expression of Twist and vimentin on CTCs using PBMC cytospin preparations from these patients. The presence of $\mathrm{CK}^{+}$cells was confirmed by immunofluorescence in all patients with metastatic disease and in 22 of 25 patients with early stage disease. Double-staining experiments with pan-CK and Twist antibodies revealed that all patients with metastatic disease had detectable double-stained cells and that CK ${ }^{+} /$Twist $^{+}$cells could be detected in $16(72.7 \%)$ of 22 patients with early stage breast cancer $(P=0.037)$ (Figure $2 \mathrm{~A}$, graph I, and Table 2). Additionally, the proportion of double-positive CTCs was lower in patients with early stage breast cancer than in patients with metastatic breast cancer (53\% versus $97 \%$, respectively; $P=0.0001$ ) (Figure 2A, graph II). Confocal laser-scanning microscopy and ARIOL system microscopy revealed that Twist was located both in the cytoplasm and in the nuclei of CTCs (Figures 2B, graph I; Figures 3 and 4; and Additional file 2). The median number of CTCs per patient expressing Twist ${ }^{+} / \mathrm{CK}^{+}$was $100 \%$ (range, $33 \%$ to $100 \%$ ) in patients with metastatic disease and $40.6 \%$ (range, $12 \%$ to $100 \%$ ) in patients with early stage breast cancer (Figure 2A, graph III). Nineteen patients with metastatic breast cancer (76\%) had only double-positive cells $\left(\mathrm{Twist}^{+} \mathrm{CK}^{+}\right.$), while in early breast cancer patients only six (27\%) of twenty-two $\mathrm{CK}^{+}$patients had exclusively Twist ${ }^{+} \mathrm{CK}^{+}$cells $(P=0.0001)$ (Table 2$)$.

We subsequently assessed the expression of vimentin in CTCs of the same cohort of patients. Vimentin was also expressed in CTCs in $100 \%$ and $77 \%$ of patients with metastatic and early stage disease, respectively $(P=$ 0.004) (Figure 2A, graph I, and Table 2). Furthermore, the proportion of double-positive CTCs was lower in patients with early stage cancer than in those with metastatic disease (56\% versus $74 \% ; P=0.005$ ) (Figure $2 \mathrm{~A}$, graph II). In addition, the median proportion of doublepositive CTCs per patient with early stage and
Table 1 Patient characteristics ${ }^{\mathrm{a}}$

\begin{tabular}{|c|c|}
\hline Early breast cancer & Metastatic breast cancer \\
\hline $\begin{array}{l}\text { Number of patients enrolled }= \\
25\end{array}$ & Number of patients enrolled $=25$ \\
\hline Age, years & Age, years \\
\hline Median (range), 59 (26 to 76) & Median (range), 59 (36 to 83) \\
\hline $\begin{array}{l}\text { ECOG performance status, } n \\
(\%)\end{array}$ & ECOG performance status, $n(\%)$ \\
\hline $024(96 \%)$ & $08(32 \%)$ \\
\hline $11(4 \%)$ & $113(52 \%)$ \\
\hline $20(0 \%)$ & $24(16 \%)$ \\
\hline Histology, n (\%) & Histology, $n(\%)$ \\
\hline Ductal 22 (88\%) & Ductal 20 (80\%) \\
\hline Lobular 3 (12\%) & Lobular 1 (4\%) \\
\hline Other $0(0 \%)$ & Unknown 4 (16\%) \\
\hline Menopausal status, n (\%) & Menopausal status, $n$ (\%) \\
\hline Premenopausal 8 (32\%) & Premenopausal 4 (16\%) \\
\hline Perimenopausal 1 (4\%) & Perimenopausal 1 (4) \\
\hline Postmenopausal 16 (64\%) & Postmenopausal 20 (80\%) \\
\hline $\begin{array}{l}\text { Hormone receptor status, } n \\
\text { (\%) }\end{array}$ & Hormone receptor status, $n(\%)$ \\
\hline $\begin{array}{l}\text { ER-positive/PR-positive } 11 \\
(44 \%)\end{array}$ & ER-positive/PR-positive 11 (44\%) \\
\hline $\begin{array}{l}\text { ER-positive/PR-negative } 6 \\
(24 \%)\end{array}$ & ER-positive/PR-negative 3 (12\%) \\
\hline ER-negative/PR-positive 1 (4\%) & ER-negative/PR-positive 2 (8\%) \\
\hline $\begin{array}{l}\text { ER-negative/PR-negative } 7 \\
(28 \%)\end{array}$ & ER-negative/PR-negative 9 (36\%) \\
\hline Tumour size, $n(\%)$ & Number of disease sites, $n(\%)$ \\
\hline 1 to $1.9 \mathrm{~cm}(\mathrm{~T} 1) 6(24 \%)$ & $17(28 \%)$ \\
\hline 2 to $5 \mathrm{~cm}(\mathrm{~T} 2) 16$ (64\%) & $29(36 \%)$ \\
\hline$>5$ cm (Т3) 1 (4\%) & $34(16 \%)$ \\
\hline Unknown 2 (8\%) & $\geq 45(20 \%)$ \\
\hline Tumour grade, $n(\%)$ & Line of treatment, $n(\%)$ \\
\hline $12(8 \%)$ & First $11(44 \%)$ \\
\hline || 9 (36\%) & Second 7 (28\%) \\
\hline III 13 (52\%) & $\geq$ Third 7 (28\%) \\
\hline \multicolumn{2}{|l|}{ Unknown 1 (4\%) } \\
\hline & $\begin{array}{l}\text { Primary breast cancer at presentation, } n \\
(\%)\end{array}$ \\
\hline & Early 9 (36\%) \\
\hline Positive nodes, $n(\%)$ & Metastatic 16 (64\%) \\
\hline \multicolumn{2}{|l|}{0 (N0) $11(44 \%)$} \\
\hline 1 to 3 (N1) $9(36 \%)$ & Visceral disease, $n(\%)$ \\
\hline 4 to 9 (N2) 2 (8\%) & Yes 13 (52\%) \\
\hline$\geq 10(\mathrm{~N} 3) 2(8 \%)$ & No 12 (48\%) \\
\hline
\end{tabular}

${ }^{\mathrm{a} E C O G}=$ Eastern Cooperative Oncology Group; $\mathrm{ER}=$ oestrogen receptor; $\mathrm{PR}=$ progesterone receptor.

metastatic disease was $56 \%$ (range, $21.8 \%$ to $100 \%$ ) and 98\% (range, $16.7 \%$ to $100 \%$ ), respectively (Figure $2 \mathrm{~A}$, graph III). In 12 (48\%) of 25 patients with metastatic disease, all the CTCs were double-positive (vimentin ${ }^{+}$ $\mathrm{CK}^{+}$) compared with only $6(27 \%)$ of 22 patients with early disease who had exclusively vimentin ${ }^{+} \mathrm{CK}^{+} \mathrm{CTCs}$ 


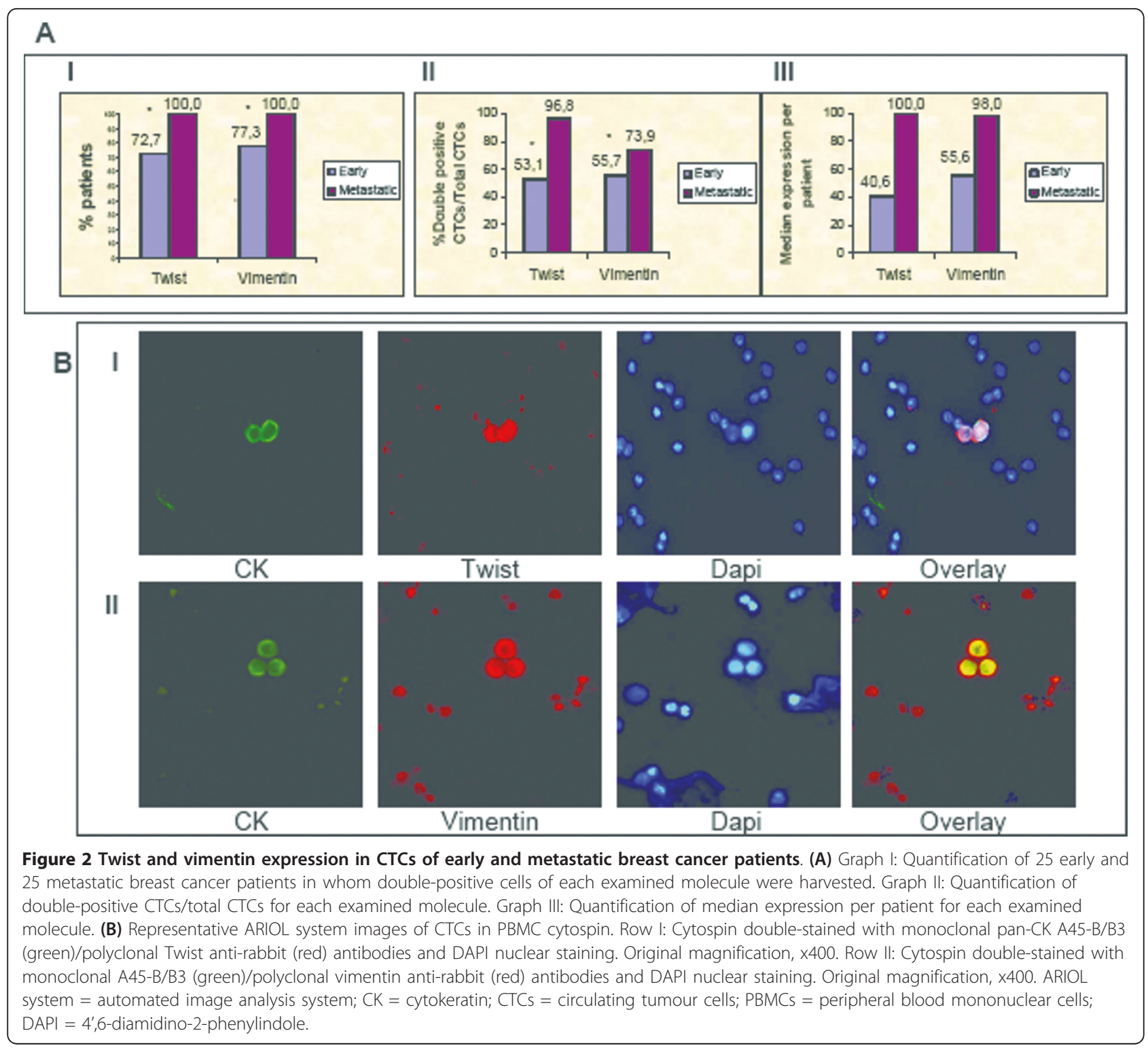

$(P=0.048)$ (Table 2$)$. Confocal laser-scanning microscopy and ARIOL system microscopy revealed that the intracellular distribution of vimentin in CTCs was almost identical to that of cytokeratin filaments (Figure 2B, graph II; Figures 3 and 4; and Additional file 2).

A statistically significant correlation (Spearman's $\rho$ analysis) was also observed between the number of CTCs expressing Twist and vimentin in early stage $(P=$ $0.027)$ and metastatic $(P=0.009)$ breast cancer patients. This correlation remained significant even when all patients were grouped together $(P=0.005)$.

Triple-immunofluorescence experiments revealed specific expression of Twist and vimentin in CTCs

As mentioned above, Twist and vimentin expression was also observed in PBMCs. To confirm that the cells characterised as CTCs in breast cancer patients were nonhematopoietic cells presenting ectopic cytokeratin expression, we performed triple-immunofluorescence experiments using antibodies against CKs and CD45 along with antibodies against Twist or vimentin. These experiments were carried out indicatively in five metastatic and eight early breast cancer patients. Our results revealed that no $\mathrm{CK}^{+}$vimentin ${ }^{+} \mathrm{CD} 45^{+}$or $\mathrm{CK}^{+} \mathrm{Twist}^{+} \mathrm{CD} 45^{+}$cells could be identified in patient samples (Figure 3 ).

\section{Vimentin and Twist are coexpressed in CTCs of breast cancer patients}

Subsequently, we investigated the possible coexpression of the examined mesenchymal markers in the same CTC. After performing CD45 immunomagnetic separation in PBMCs isolated from 24 patients with metastatic 
Table 2 Number of double-stained CTCs/250,000 PBMCs in early and metastatic breast cancer patients ${ }^{a}$

\begin{tabular}{|c|c|c|c|c|c|c|c|c|c|}
\hline \multicolumn{5}{|c|}{ Early breast cancer } & \multicolumn{5}{|c|}{ Metastatic breast cancer } \\
\hline Patient & $\operatorname{Vim}^{+} \mathrm{CK}^{+}$ & $\operatorname{Vim}^{-} \mathrm{CK}^{+}$ & Twist $^{+} \mathrm{CK}^{+}$ & Twist $^{-} \mathrm{CK}^{+}$ & Patient & $\operatorname{Vim}^{+} \mathrm{CK}^{+}$ & $\operatorname{Vim}^{-} \mathrm{CK}^{+}$ & Twist $^{+} \mathrm{CK}^{+}$ & Twist $^{-} \mathrm{CK}^{+}$ \\
\hline 1 & 64 & 51 & 83 & 100 & 1 & 5 & 0 & 4 & 0 \\
\hline 2 & 25 & 63 & 29 & 142 & 2 & 45 & 0 & 8 & 0 \\
\hline 3 & 31 & 111 & 578 & 68 & 3 & 21 & 0 & 7 & 0 \\
\hline 4 & 35 & 28 & 53 & 85 & 4 & 2 & 0 & 4 & 0 \\
\hline 5 & 135 & 199 & 18 & 134 & 5 & 74 & 20 & 45 & 0 \\
\hline 6 & 100 & 158 & 28 & 170 & 6 & 7 & 7 & 3 & 6 \\
\hline 7 & 170 & 254 & 89 & 423 & 7 & 7 & 2 & 420 & 0 \\
\hline 8 & 0 & 0 & 74 & 4 & 8 & 21 & 69 & 2 & 0 \\
\hline 9 & 0 & 1 & 71 & 95 & 9 & 10 & 90 & 3 & 0 \\
\hline 10 & 140 & 4 & 0 & 1 & 10 & 2 & 0 & 2 & 0 \\
\hline 11 & 22 & 0 & 0 & 0 & 11 & 3 & 15 & 103 & 36 \\
\hline 12 & 17 & 0 & 74 & 88 & 12 & 67 & 3 & 9 & 1 \\
\hline 13 & 0 & 4 & 8 & 0 & 13 & 4 & 0 & 0 & 0 \\
\hline 14 & 0 & 0 & 0 & 3 & 14 & 3 & 3 & 42 & 0 \\
\hline 15 & 7 & 1 & 0 & 4 & 15 & 1 & 0 & 1 & 0 \\
\hline 16 & 10 & 3 & 12 & 0 & 16 & 1 & 0 & 1 & 0 \\
\hline 17 & 0 & 0 & 0 & 0 & 17 & 10 & 2 & 55 & 0 \\
\hline 18 & 28 & 12 & 0 & 0 & 18 & 325 & 97 & 5 & 7 \\
\hline 19 & 0 & 0 & 0 & 10 & 19 & 17 & 1 & 13 & 0 \\
\hline 20 & 0 & 1 & 2 & 0 & 20 & 3 & 0 & 0 & 0 \\
\hline 21 & 2 & 0 & 13 & 0 & 21 & 2 & 0 & 2 & 0 \\
\hline 22 & 2 & 0 & 0 & 9 & 22 & 2 & 1 & 2 & 0 \\
\hline 23 & 306 & 0 & 380 & 0 & 23 & 9 & 0 & 658 & 0 \\
\hline 24 & 1 & 0 & 0 & 2 & 24 & 192 & 4 & 44 & 0 \\
\hline 25 & 24 & 0 & 1 & 0 & 25 & 40 & 0 & 80 & 0 \\
\hline
\end{tabular}

${ }^{\mathrm{a}} \mathrm{CTCs}=$ circulating tumour cells; PBMCs = peripheral blood mononuclear cells; Vim = vimentin; CK = cytokeratin.

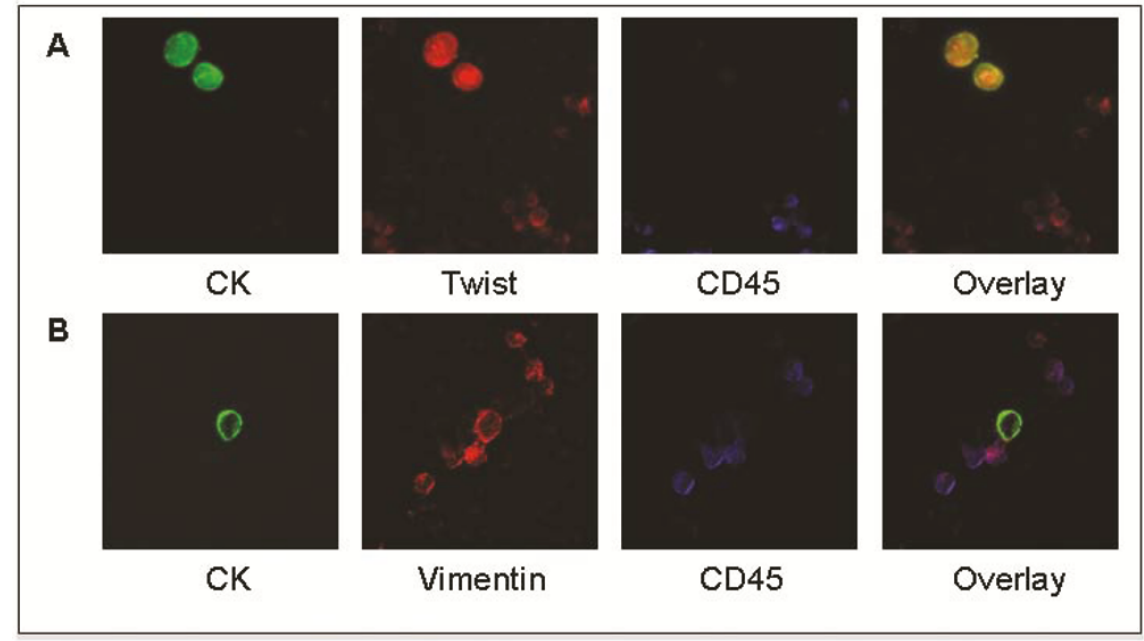

Figure 3 Triple-immunofluorescence (CK/Twist/CD45, CK/vimentin/CD45) in CTCs. Representative ARIOL system photomicrographs of CTC cytospin after negative immunomagnetic separation in patients with metastatic breast cancer. (A) Cells were triple-stained with pan-CK A45-B/B3 antibody/Zenon Alexa Fluor 488 (green)/Twist anti-rabbit antibody/Alexa Fluor 555 anti-rabbit antibody (orange) and CD45 anti-mouse/Alexa Fluor 633 anti-mouse antibody (blue). Original magnification, x400. (B) Cells were triple-stained with pan-CK A45-B/B3 antibody/Zenon-Alexa Fluor 488 (green)/vimentin anti-rabbit antibody/Alexa Fluor 555 anti-rabbit antibody (orange) and CD45 anti-mouse/Alexa Fluor 633 anti-mouse antibody (blue). Original magnification, $x 400$. ARIOL system = automated image analysis system; CK = cytokeratin; CTCs = circulating tumour cells. 


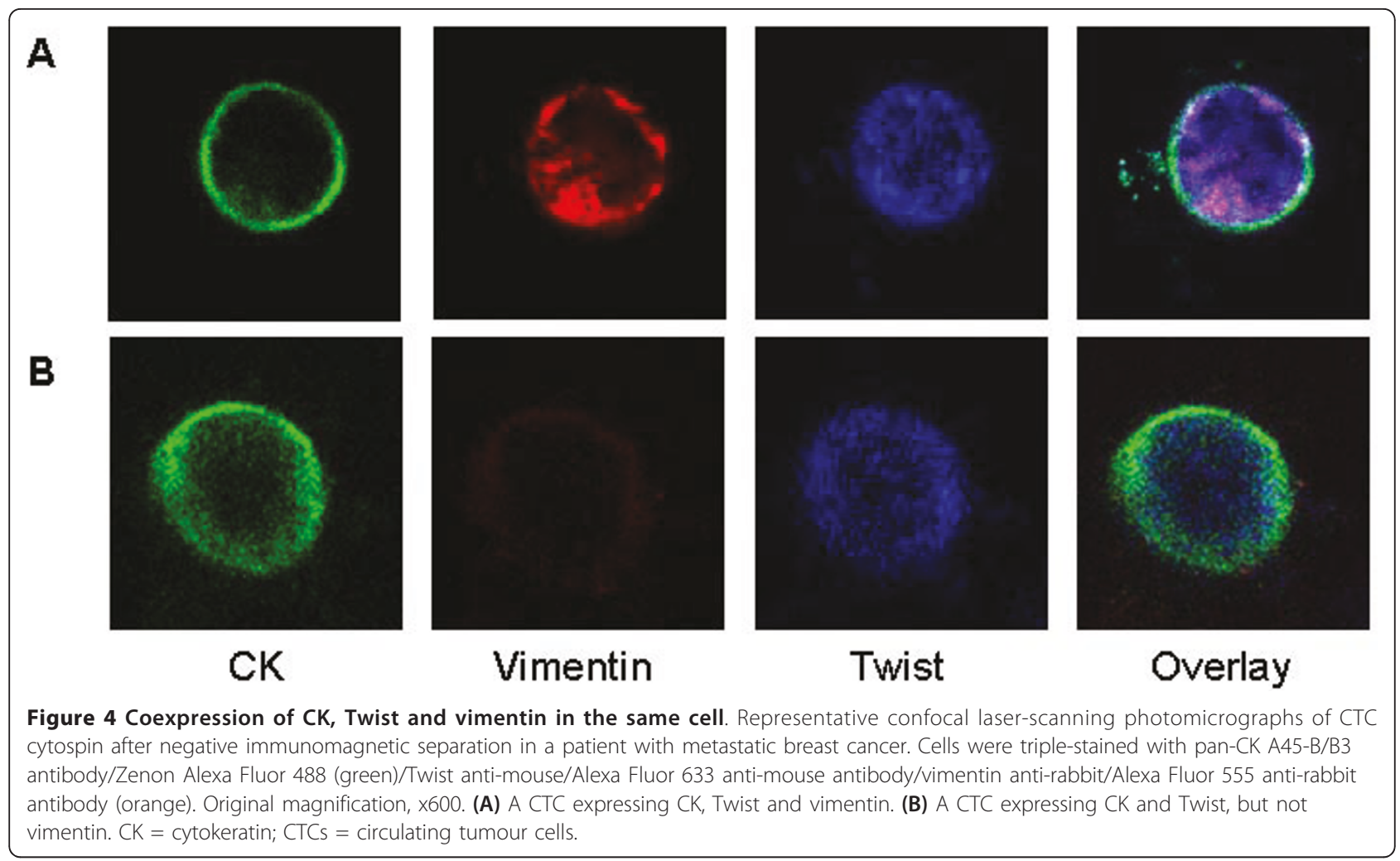

disease, the CTC-enriched fraction was cytocentrifuged on one slide for each patient. Triple-immunofluorescence experiments using anti-pan-CK, anti-Twist and anti-vimentin antibodies revealed that 16 (64\%) of 25 CTCs identified were $\mathrm{CK}^{+}$Twist $^{+}$vimentin ${ }^{+}$and 9 (36\%) of 25 CTCs identified were $\mathrm{CK}^{+}$Twist $^{+}$vimentin ${ }^{-}$. None of the detected CTCs had the $\mathrm{CK}^{+} \mathrm{Tw}_{\text {wist }}{ }^{-}$vimentin ${ }^{+}$phenotype. These results indicate that Twist and vimentin are coexpressed in a subset of CTCs of metastatic breast cancer patients (Figure 4).

\section{Discussion}

There is increasing evidence that the presence of CTCs and DTCs is correlated with minimal residual disease or disease progression in patients with breast cancer. Nevertheless, the underlying molecular characteristics of micrometastatic cells associated with the development of overt metastases remain largely unknown. EMT is a multistep process that has been suggested to play a key role in cancer progression and metastasis [12]. Accordingly, CTCs bearing characteristics of an EMT phenotype should be actively involved in tumour dissemination, proliferation and metastasis. Twist is a transcription factor that, among others, participates in EMT and is upregulated in many tumour cells [18-22,47]. In a recent report by Watson et al. [24], Twist expression was specifically enhanced in a gene signature obtained from epithelial cell adhesion molecule-enriched bone marrow samples of patients with breast cancer after neoadjuvant chemotherapy. Twist also increases VEGF expression, while it is directly regulated by HIF-1 $\alpha[30,31]$. Since we have recently shown that $62 \%$ and $76 \%$ of breast cancer patients express VEGF and HIF-1 $\alpha$, respectively, in their CTCs [33], it was of interest to identify the potential expression of this transcription factor in CTCs.

In the present cytomorphological study, we compared for the first time the expression of Twist and vimentin in individual CTCs of patients with early stage versus metastatic breast cancer. For this purpose, we performed double-staining experiments with the corresponding antibodies in PBMC cytospin preparations of 25 early stage and 25 metastatic breast cancer patients. To confirm the specificity of our results, we also performed triple-staining experiments with pan-CK/Twist/CD45 and pan-CK/vimentin/CD45 antibodies, which revealed that the hematopoietic antigen CD45 was not expressed in $\mathrm{CK}^{+} / \mathrm{Twist}^{+}$or $\mathrm{CK}^{+} /$vimentin ${ }^{+}$cells. These findings clearly indicate that the observed expression of Twist and vimentin is not confined to hematopoietic cells.

Double-staining experiments revealed that Twist was expressed in all CK-19 mRNA $^{+}$metastatic patients, while most of them (76\%) had exclusively double-positive cells $\left(\mathrm{CK}^{+} \mathrm{Twist}^{+}\right)$. The median expression per 
patient of Twist in CTCs was also high at 100\%. Similar results have also been reported in two recent studies by Aktas et al. [48] and Mego et al. [49]. In these studies, EMT markers including Twist were detected in CTCs of breast cancer patients at lower percentages of $42 \%$.and $57.7 \%$, respectively. This lower level of expression might be related to the difference in the identification method or to immunomagnetic enrichment of tumour cells.

Interestingly, when vimentin expression was investigated in the same cohort of patients, it was observed that, similarly to Twist, vimentin was expressed in CTCs of all the evaluated patients with metastatic disease, and the proportion of patients who had exclusively doublepositive cells $\left(\mathrm{CK}^{+}\right.$vimentin $\left.{ }^{+}\right)$was also high. The median percentage of vimentin-expressing CTCs per patient was $98 \%$. Vimentin is an intermediate filament of mesenchymal cells that is commonly used to identify cells undergoing EMT in cancer. In addition, its expression is associated with increased risk of metastasis and poor prognosis in breast cancer patients $[37,38]$. There are only two previous reports verifying the expression of vimentin in DTC cell lines and in CTCs $[38,50]$. In agreement with these data, the high rate of Twist and vimentin expression in CTCs observed in our study implies that mesenchymal markers are highly expressed in CTCs of metastatic breast cancer patients and denotes that the majority of these cells are undergoing the EMT process.

Furthermore, our experiments revealed that $\mathrm{CK}^{+} \mathrm{T}$ wist ${ }^{+}$and $\mathrm{CK}^{+}$vimentin ${ }^{+} \mathrm{CTCs}$ were also observed in patients with early stage breast cancer. This observation suggests that circulating epithelial $\left(\mathrm{CK}^{+}\right)$cells with an EMT phenotype may be involved in the continuous tumour spreading in patients with clinically undetectable metastases. However, the EMT phenotype expression was significantly lower $(P=0.037$ for Twist and $P=$ 0.004 for vimentin) in early stage cancer patients than in metastatic breast cancer patients. The median expression of Twist and vimentin was also significantly lower (40.6\% and $55.6 \%$, respectively) in early stage cancer patients, but it was extremely variable from patient to patient (ranges, $11 \%$ to $100 \%$ and $21 \%$ to $100 \%$, respectively). The proportion of double-positive CTCs in early stage cancer patients was $53 \%$ and $56 \%$ for Twist and vimentin, respectively, which was also significantly different from metastatic breast cancer patients $(P=$ 0.0001 and $P=0.05$, respectively). These findings clearly indicate that EMT markers may be expressed in CTCs of patients with early stage disease, but to a lesser extent than in patients with metastatic disease. In addition, the above results imply that the population of CTCs expressing EMT markers predominates during disease progression or, alternatively, this cell population is selected over non-EMT-expressing CTCs because of resistance to therapy. However, this finding should be interpreted with caution because of the smaller number of CTCs detected in patients with early stage compared to advanced disease, which might influence the chance to find an EMT-like CTC.

A statistically significant correlation between the number of CTCs expressing Twist and vimentin in patients with both early stage and metastatic disease was demonstrated $(P=0.005$ and $P=0.027$, respectively), suggesting that both biomarkers were simultaneously expressed in CTCs. This assumption was confirmed by triplestaining experiments using antibodies against CK, Twist and vimentin in immunomagnetically isolated CTCs from 24 patients, which demonstrated that the majority (64\%) of CTCs had the $\mathrm{CK}^{+}$Twist $^{+}$vimentin ${ }^{+}$phenotype. Nevertheless, $36 \%$ of the CTCs were $\mathrm{CK}^{+}$Twist $^{+}$vimentin $^{-}$, whereas there were no cells with the $\mathrm{CK}^{+}$Twistvimentin $^{+}$phenotype (Figure 4). These findings indicate the heterogeneity of CTCs. It is also possible that Twist expression is a more common phenomenon during EMT, thus suggesting Twist as a more specific marker for EMT.

Finally, a differential intracellular distribution for vimentin and Twist was observed in CTCs. Vimentin was primarily located in the cytoplasm, similarly to the intracellular distribution of CK (Figures 2, 3, 4). This observation is in agreement with the findings of previous reports showing that vimentin filaments follow the preexisting CK network during EMT progression [51]. Twist showed both cytoplasmic and nuclear localisation as expected, considering its function as a transcription factor (Figures 2, 3, 4).

\section{Conclusions}

The results of the present study clearly indicate that EMT markers such as Twist and vimentin are expressed in CTCs of patients with early stage and metastatic breast cancer. The variable expression of these molecules in CTCs at different stages of disease implies the predominance of the EMT phenotype during disease evolution. This hypothesis is further supported by the observation that $\mathrm{CK}^{+} \mathrm{CTCs}$ in patients with early stage breast cancer are more heterogeneous with regard to the expression of EMT markers, necessitating additional studies to further elucidate their distinct biological role.

\section{Additional material}

\footnotetext{
Additional file 1: Triple-staining control experiments in HeLa cells Triple-staining control experiments in HeLa cells analysed using a confocal laser-scanning microscope. (I) Representative confocal laserscanning photomicrographs of positive controls for Twist anti-mouse antibody. HeLa cells were stained with pan-CK A45-B/B3 antibody/Zenon Alexa Fluor 488 (green)/Twist anti-mouse/Alexa Fluor 633 anti-mouse IgG (blue)/vimentin anti-rabbit antibody/Alexa Fluor 555 anti-rabbit antibody
} 
(red). Original magnification, x500. (II) Negative control for Twist. HeLa cells stained with pan-CK A45-B/B3 antibody/Zenon Alexa Fluor 488 (green)/Alexa Fluor 633 anti-mouse lgG (blue)/vimentin anti-rabbit antibody/Alexa Fluor 555 anti-rabbit antibody (red). Original magnification, x500. (II) Negative control for vimentin. HeLa cells were stained with pan-CK A45-B/B3 antibody/Zenon Alexa Fluor 488 (green)/ Twist anti-mouse antibody/Alexa Fluor 633 anti-mouse antibody (blue)/ Alexa Fluor $555 \mathrm{lgG}$ isotype antibody (red). Original magnification, x500. CK = cytokeratin; HeLa = patient with cervical adenocarcinoma, from which the cell line was derived; lgG, immunoglobulin G.

Additional file 2: Twist and vimentin expression in CTCs of breast cancer patients. Representative confocal laser-scanning microscopic images of Twist-or vimentin-expressing CTCS of breast cancer patients. (I) Representative confocal laser-scanning microscopic images of breast cancer patients' cytospin double-stained with monoclonal pan-CK (A45B/B3)/Alexa Fluor 488 anti-mouse antibody (green)/polyclonal Twist antirabbit antibody and Alexa Fluor 555 anti-rabbit antibody (red). Original magnification, $\times 600$. (II) Representative images of cytospin double-stained with monoclonal pan-CK (A45-B/B3)/Alexa Fluor 488 anti-mouse antibody (green)/polyclonal vimentin anti-rabbit antibody and Alexa Fluor 555 anti-rabbit antibody (red). Original magnification, $x 600$. CK = cytokeratin; $\mathrm{CTCS}=$ circulating tumour cells; FITC = fluorescein isothiocyanate.

\section{Abbreviations}

ARIOL system: automated image analysis system; BSA: bovine serum albumin; CTCs: circulating tumour cells; DMEM: Dulbecco's modified Eagle's medium; DTCs: disseminated tumour cells; ECOG: Eastern Cooperative Oncology Group; EMT: epithelial-to-mesenchymal transition; FITC: fluorescein isothiocyanate; HeLa: patient with cervical adenocarcinoma from which the cell line was derived; PBMCs: peripheral blood mononuclear cells; PBS: phosphate-buffered saline; RT-PCR: reverse transcriptase polymerase chain reaction.

\section{Acknowledgements}

This work was supported by Research Grants from Cretan Association of Biomedical Research (CABR).

\section{Author details}

'Laboratory of Tumor Cell Biology, University of Crete, Voutes 71110, Heraklion, Crete, Greece. ${ }^{2}$ Department of Medical Oncology, University of Crete, Voutes 71110, Heraklion, Crete, Greece.

\section{Authors' contributions}

GK participated in the design and coordination of the study, performed the immunomagnetic separations and the cell cultures and drafted the manuscript. MP and EP performed the immunofluorescence experiments and were involved in drafting the manuscript. SA helped draft the manuscript and collected all the clinicopathological characteristics of the patients. DM helped to draft the manuscript and participated in study design. VG provided general support, participated in study design and was involved in drafting the manuscript. All the authors gave their final approval of the version to be published.

\section{Competing interests}

The authors declare that they have no competing interests.

Received: 6 December 2010 Revised: 10 May 2011

Accepted: 10 June 2011 Published: 10 June 2011

\section{References}

1. Braun S, Naume B: Circulating and disseminated tumor cells. J Clin Oncol 2005, 23:1623-1626.

2. Pantel $K$, Müller V, Auer M, Nusser N, Harbeck N, Braun S: Detection and clinical implications of early systemic tumor cell dissemination in breast cancer. Clin Cancer Res 2003, 9:6326-6334.

3. Lobodasch $K$, Fröhlich F, Rengsberger M, Schubert R, Dengler R, Pachmann U, Pachmann K: Quantification of circulating tumour cells for the monitoring of adjuvant therapy in breast cancer: an increase in cell number at completion of therapy is a predictor of early relapse. Breast 2007, 16:211-218.

4. Stathopoulou A, Vlachonikolis I, Mavroudis D, Perraki M, Kouroussis C, Apostolaki S, Malamos N, Kakolyris S, Kotsakis A, Xenidis N, Reppa D, Georgoulias V: Molecular detection of cytokeratin-19-positive cells in the peripheral blood of patients with operable breast cancer: evaluation of their prognostic significance. J Clin Oncol 2002, 20:3404-3412.

5. Xenidis N, Perraki M, Kafousi M, Apostolaki S, Bolonaki I, Stathopoulou A Kalbakis K, Androulakis N, Kouroussis C, Pallis T, Christophylakis C, Argyraki K, Lianidou ES, Stathopoulos S, Georgoulias V, Mavroudis D: Predictive and prognostic value of peripheral blood cytokeratin-19 mRNA-positive cells detected by real-time polymerase chain reaction in node-negative breast cancer patients. J Clin Oncol 2006, 24:3756-3762.

6. Cristofanilli M, Budd GT, Ellis MJ, Stopeck A, Matera J, Miller MC, Reuben JM, Doyle GV, Allard WJ, Terstappen LW, Hayes DF: Circulating tumor cells, disease progression, and survival in metastatic breast cancer. N Engl J Med 2004, 351:781-791.

7. Cristofanilli M, Hayes DF, Budd GT, Ellis MJ, Stopeck A, Reuben JM, Doyle GV, Matera J, Allard WJ, Miller MC, Fritsche HA, Hortobagyi GN, Terstappen LW: Circulating tumor cells: a novel prognostic factor for newly diagnosed metastatic breast cancer. J Clin Oncol 2005, 23:1420-1430.

8. Bidard FC, Vincent-Salomon A, Sigal-Zafrani B, Diéras V, Mathiot C, Mignot L, Thiery JP, Sastre-Garau X, Pierga JY: Prognosis of women with stage IV breast cancer depends on detection of circulating tumor cells rather than disseminated tumor cells. Ann Oncol 2008, 19:496-500.

9. Bozionellou V, Mavroudis D, Perraki M, Papadopoulos S, Apostolaki S, Stathopoulos E, Stathopoulou A, Lianidou E, Georgoulias V: Trastuzumab administration can effectively target chemotherapy-resistant cytokeratin19 messenger RNA-positive tumor cells in the peripheral blood and bone marrow of patients with breast cancer. Clin Cancer Res 2004, 10:8185-8194.

10. Fehm T, Sagalowsky A, Clifford E, Beitsch P, Saboorian H, Euhus D, Meng S, Morrison L, Tucker T, Lane N, Ghadimi BM, Heselmeyer-Haddad K, Ried T, Rao C, Uhr J: Cytogenetic evidence that circulating epithelial cells in patients with carcinoma are malignant. Clin Cancer Res 2002, 8:2073-2084.

11. Klein CA, Blankenstein TJ, Schmidt-Kittler O, Petronio M, Polzer B, Stoecklein NH, Riethmüller G: Genetic heterogeneity of single disseminated tumour cells in minimal residual cancer. Lancet 2002, 360:683-689.

12. Paterlini-Brechot P, Benali NL: Circulating tumor cells (CTC) detection: clinical impact and future directions. Cancer Lett 2007, 253:180-204.

13. Guarino M: Epithelial-mesenchymal transition and tumour invasion. Int J Biochem Cell Biol 2007, 39:2153-2160.

14. Behrens J: Cadherins and catenins: role in signal transduction and tumor progression. Cancer Metastasis Rev 1999, 18:15-30.

15. Cano A, Pérez-Moreno MA, Rodrigo I, Locascio A, Blanco MJ, del Barrio MG, Portillo F, Nieto MA: The transcription factor snail controls epithelialmesenchymal transitions by repressing E-cadherin expression. Nat Cell Biol 2000, 2:76-83.

16. Nakajima S, Doi R, Toyoda E, Tsuji S, Wada M, Koizumi M, Tulachan SS, Ito D, Kami K, Mori T, Kawaguchi Y, Fujimoto K, Hosotani R, Imamura M: Ncadherin expression and epithelial-mesenchymal transition in pancreatic carcinoma. Clin Cancer Res 2004, 10:4125-4133.

17. Vesuna F, van Diest P, Chen JH, Raman V: Twist is a transcriptional repressor of E-cadherin gene expression in breast cancer. Biochem Biophys Res Commun 2008, 367:235-241.

18. Entz-Werlé N, Stoetzel C, Berard-Marec P, Kalifa C, Brugiere L, Pacquement H, Schmitt C, Tabone MD, Gentet JC, Quillet R, Oudet P, Lutz P, Babin-Boilletot A, Gaub MP, Perrin-Schmitt F: Frequent genomic abnormalities at TWIST in human pediatric osteosarcomas. Int I Cancer 2005, 117:349-355.

19. Hoek K, Rimm DL, Williams KR, Zhao H, Ariyan S, Lin A, Kluger HM, Berger AJ, Cheng E, Trombetta ES, Wu T, Niinobe M, Yoshikawa K, Hannigan $G E$, Halaban R: Expression profiling reveals novel pathways in the transformation of melanocytes to melanomas. Cancer Res 2004, 64:5270-5282.

20. Kwok WK, Ling MT, Lee TW, Lau TC, Zhou C, Zhang X, Chua CW, Chan KW, Chan FL, Glackin C, Wong YC, Wang X: Up-regulation of TWIST in prostate cancer and its implication as a therapeutic target. Cancer Res 2005, 65:5153-5162. 
21. Rosivatz E, Becker I, Specht K, Fricke E, Luber B, Busch R, Höfler H, Becker KF: Differential expression of the epithelial-mesenchymal transition regulators Snail, SIP1, and Twist in gastric cancer. Am J Pathol 2002, 161:1881-1891.

22. van Doorn R, Dijkman R, Vermeer MH, Out-Luiting JJ, van der RaaijHelmer EM, Willemze R, Tensen CP: Aberrant expression of the tyrosine kinase receptor EphA4 and the transcription factor twist in Sézary syndrome identified by gene expression analysis. Cancer Res 2004, 64:5578-5586.

23. Watanabe O, Imamura H, Shimizu T, Kinoshita J, Okabe T, Hirano A, Yoshimatsu K, Konno S, Aiba M, Ogawa K: Expression of Twist and Wnt in human breast cancer. Anticancer Res 2004, 24:3851-3856.

24. Watson MA, Ylagan LR, Trinkaus KM, Gillanders WE, Naughton MJ, Weilbaecher KN, Fleming TP, Aft RL: Isolation and molecular profiling of bone marrow micrometastases identifies TWIST1 as a marker of early tumor relapse in breast cancer patients. Clin Cancer Res 2007, 13:5001-5009.

25. Bonnomet A, Brysse A, Tachsidis A, Waltham M, Thompson EW, Polette M, Gilles C: Epithelial-to-mesenchymal transitions and circulating tumor cells. J Mammary Gland Biol Neoplasia 2010, 15:261-273.

26. Cheng GZ, Chan J, Wang Q, Zhang W, Sun CD, Wang LH: Twist transcriptionally up-regulates AKT2 in breast cancer cells leading to increased migration, invasion, and resistance to paclitaxel. Cancer Res 2007, 67:1979-1987.

27. Wang X, Ling MT, Guan XY, Tsao SW, Cheung HW, Lee DT, Wong YC: Identification of a novel function of TWIST, a bHLH protein, in the development of acquired taxol resistance in human cancer cells. Oncogene 2004, 23:474-482.

28. Zhang $X$, Wang $Q$, Ling MT, Wong YC, Leung SC, Wang X: Anti-apoptotic role of TWIST and its association with Akt pathway in mediating taxol resistance in nasopharyngeal carcinoma cells. Int J Cancer 2007, 120:1891-1898.

29. Mironchik Y, Winnard PT Jr, Vesuna F, Kato Y, Wildes F, Pathak AP, Kominsky S, Artemov D, Bhujwalla Z, Van Diest P, Burger H, Glackin C, Raman $V$ : Twist overexpression induces in vivo angiogenesis and correlates with chromosomal instability in breast cancer. Cancer Res 2005, 65:10801-10809

30. Yang MH, Wu KJ: TWIST activation by hypoxia inducible factor-1 (HIF-1): implications in metastasis and development. Cell Cycle 2008, 7:2090-2096.

31. Yang MH, Wu MZ, Chiou SH, Chen PM, Chang SY, Liu CJ, Teng SC, Wu KJ: Direct regulation of TWIST by HIF-1a promotes metastasis. Nat Cell Biol 2008, 10:295-305.

32. Kallergi G, Agelaki S, Kalykaki A, Stournaras C, Mavroudis D, Georgoulias V: Phosphorylated EGFR and PI3K/Akt signaling kinases are expressed in circulating tumor cells of breast cancer patients. Breast Cancer Res 2008, 10:R80.

33. Kallergi G, Markomanolaki H, Giannoukaraki V, Papadaki MA, Strati A Lianidou ES, Georgoulias V, Mavroudis D, Agelaki S: Hypoxia-inducible factor-1a and vascular endothelial growth factor expression in circulating tumor cells of breast cancer patients. Breast Cancer Res 2009, 11:R84.

34. Franke WW, Grund C, Kuhn C, Jackson BW, Illmensee K: Formation of cytoskeletal elements during mouse embryogenesis. III. Primary mesenchymal cells and the first appearance of vimentin filaments. Differentiation 1982, 23:43-59.

35. Mclnroy L, Määttä A: Down-regulation of vimentin expression inhibits carcinoma cell migration and adhesion. Biochem Biophys Res Commun 2007, 360:109-114.

36. Zhao $Y$, Yan Q, Long $X$, Chen $X$, Wang Y: Vimentin affects the mobility and invasiveness of prostate cancer cells. Cell Biochem Funct 2008 , 26:571-577.

37. Domagala W, Lasota J, Bartkowiak J, Weber K, Osborn M: Vimentin is preferentially expressed in human breast carcinomas with low estrogen receptor and high Ki-67 growth fraction. Am J Pathol 1990, 136:219-227.

38. Thomas PA, Kirschmann DA, Cerhan JR, Folberg R, Seftor EA, Sellers TA, Hendrix MJ: Association between keratin and vimentin expression, malignant phenotype, and survival in postmenopausal breast cancer patients. Clin Cancer Res 1999, 5:2698-2703.

39. Willipinski-Stapelfeldt B, Riethdorf S, Assmann V, Woelfle U, Rau T, Sauter G, Heukeshoven J, Pantel K: Changes in cytoskeletal protein composition indicative of an epithelial-mesenchymal transition in human micrometastatic and primary breast carcinoma cells. Clin Cancer Res 2005, 11:8006-8014.

40. Bartkowiak K, Wieczorek M, Buck F, Harder S, Moldenhauer J, Effenberger KE, Pantel K, Peter-Katalinic J, Brandt BH: Two-dimensional differential gel electrophoresis of a cell line derived from a breast cancer micrometastasis revealed a stem/progenitor cell protein profile. $J$ Proteome Res 2009, 8:2004-2014.

41. Ring $A$, Smith $I E$, Dowsett M: Circulating tumour cells in breast cancer. Lancet Oncol 2004, 5:79-88.

42. Stathopoulou A, Gizi A, Perraki M, Apostolaki S, Malamos N, Mavroudis D, Georgoulias V, Lianidou ES: Real-time quantification of CK-19 mRNApositive cells in peripheral blood of breast cancer patients using the lightcycler system. Clin Cancer Res 2003, 9:5145-5151.

43. Kallergi G, Mavroudis D, Georgoulias V, Stournaras C: Phosphorylation of FAK, PI-3K, and impaired actin organization in CK-positive micrometastatic breast cancer cells. Mol Med 2007, 13:79-88.

44. Meng S, Tripathy D, Frenkel EP, Shete S, Naftalis EZ, Huth JF, Beitsch PD, Leitch M, Hoover S, Euhus D, Haley B, Morrison L, Fleming TP, Herlyn D, Terstappen LW, Fehm T, Tucker TF, Lane N, Wang J, Uhr JW: Circulating tumor cells in patients with breast cancer dormancy. Clin Cancer Res 2004, 10:8152-8162.

45. Fehm T, Solomayer EF, Meng S, Tucker T, Lane N, Wang J, Gebauer G: Methods for isolating circulating epithelial cells and criteria for their classification as carcinoma cells. Cytotherapy 2005, 7:171-185.

46. Naume B, Borgen E, Nesland JM, Beiske K, Gilen E, Renolen A, Ravnås G, Qvist H, Kåresen R, Kvalheim G: Increased sensitivity for detection of micrometastases in bone-marrow/peripheral-blood stem-cell products from breast-cancer patients by negative immunomagnetic separation. Int J Cancer 1998, 78:556-560.

47. Yang J, Mani SA, Donaher JL, Ramaswamy S, Itzykson RA, Come C, Savagner P, Gitelman I, Richardson A, Weinberg RA: Twist, a master regulator of morphogenesis, plays an essential role in tumor metastasis. Cell 2004, 117:927-939.

48. Aktas B, Tewes M, Fehm T, Hauch S, Kimmig R, Kasimir-Bauer S: Stem cell and epithelial-mesenchymal transition markers are frequently overexpressed in circulating tumor cells of metastatic breast cancer patients. Breast Cancer Res 2009, 11:R46.

49. Mego M, Mani SA, Lee BN, Li C, Evans KW, Cohen EN, Gao H, Jackson SA, Giordano A, Hortobagyi GN, Cristofanilli M, Lucci A, Reuben JM: Expression of epithelial-mesenchymal transition-inducing transcription factors in primary breast cancer: the effect of neoadjuvant therapy. Int $J$ Cancer .

50. Raimondi C, Gradilone A, Naso G, Vincenzi B, Petracca A, Nicolazzo C, Palazzo A, Saltarelli R, Spremberg F, Cortesi E, Gazzaniga P: Epithelialmesenchymal transition and stemness features in circulating tumor cells from breast cancer patients. Breast Cancer Res Treat

51. Pagan R, Martín I, Alonso A, Llobera M, Vilaró S: Vimentin filaments follow the preexisting cytokeratin network during epithelial-mesenchymal transition of cultured neonatal rat hepatocytes. Exp Cell Res 1996, 222:333-344

doi:10.1186/bcr2896

Cite this article as: Kallergi et al:: Epithelial to mesenchymal transition markers expressed in circulating tumour cells of early and metastatic breast cancer patients. Breast Cancer Research 2011 13:R59.

\section{Submit your next manuscript to BioMed Central and take full advantage of:}

- Convenient online submission

- Thorough peer review

- No space constraints or color figure charges

- Immediate publication on acceptance

- Inclusion in PubMed, CAS, Scopus and Google Scholar

- Research which is freely available for redistribution

Submit your manuscript at www.biomedcentral.com/submit
C Biomed Central 